\title{
PRESPECTRA AND TOWERS OVER MODEL CATEGORIES
}

\author{
KAJ MALM
}

\section{Introduction}

In [2] Edwards and Hastings have given a simple closed model category structure for tow- $\mathscr{C}$, the category of towers of a closed model category $\mathscr{C}$. A similar - duly dualized - structure can be defined for Ps $\mathscr{C}$, the category of prespectra over $\mathscr{C}$, where $\mathscr{C}$ is a closed model category equipped with an endofunctor $S$ which in some sense is compatible with the model structure. We shall prove the following: If $S$ has a right adjoint, there is an adjoint couple Ps $\left(\mathscr{C}^{\circ}\right) \approx(\text { tow- } \mathscr{C})^{\circ}$ (where ${ }^{\circ}$ denotes dualization), which under weak conditions induces an adjoint couple Ho Ps $\left(\mathscr{C}^{\circ}\right) \rightleftharpoons$ Ho $(\text { tow }-\mathscr{C})^{\circ}$. This result has bearing on stable homotopy theory, as the homotopy theory of Ps $\mathscr{C}$ is that of a stabilized category ([1], [6]).

\section{A model category structure for tow- $\mathscr{C}$}

Let $\mathscr{C}$ be a closed model category and tow- $\mathscr{C}$ the category whose objects are diagrams

$$
X: \ldots \rightarrow X_{n} \rightarrow X_{n-1} \rightarrow \ldots \rightarrow X_{1} \rightarrow X_{0}
$$

in $\mathscr{C}$ and the morphisms $f: X \rightarrow Y$ of which are commutative diagrams

in $\mathscr{C}$.

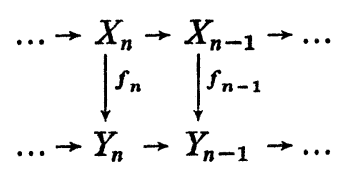

1.1. Definition. A morphism $f: X \rightarrow Y$ in tow- $\mathscr{C}$ is a

i) weak equivalence if every $f_{n}: X_{n} \rightarrow Y_{n}$ is a weak equivalence,

ii) cofibration if every $f_{n}$ is a cofibration, 
iii) fibration if every $f_{n}$ is a fibration and the induced morphism in the diagram

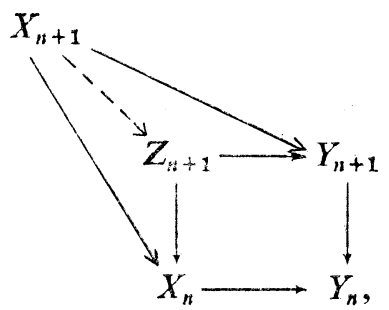

where $Z_{n+1}$ is the pullback, is a fibration for every $n \in \mathbf{N}$.

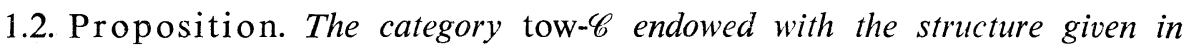
1.1 is a closed model category.

Proof. Cf. [2], 3.2.2 and 3.2.7.

\section{Prespectra over a closed model category}

Let $\mathscr{C}$ be a closed model category, $S: \mathscr{C} \rightarrow \mathscr{C}$ a functor. A (positive) prespectrum over $\mathscr{C}$ is a sequence of objects $\left(C_{n}\right)_{n \in \mathbf{N}}$ together with morphisms $S C_{n} \rightarrow C_{n+1}$; morphisms between prespectra are sequences of morphisms in $\mathscr{C}$ with the usual commutativity condition. The category of such prespectra is denoted by Ps $\mathscr{C}$.

2.1. Proposition. Suppose $S$ preserves cofibrations, trivial cofibrations and finite colimits. Then Ps $\mathscr{C}$ is a closed model category if a morphism $f:\left(C_{n}\right) \rightarrow\left(C_{n}^{\prime}\right)$ is defined to be a

i) weak equivalence, if every $f_{n}: C_{n} \rightarrow C_{n}^{\prime}$ is a weak equivalence in $\mathscr{C}$,

ii) fibration, if every $f_{n}$ is a fibration,

iii) cofibration, if every $f_{n}$ is a cofibration and the induced morphism $j_{n+1}: D_{n+1} \rightarrow C_{n+1}^{\prime}$ in the following pushout diagram is a cofibration for every $n \in \mathbf{N}$ :

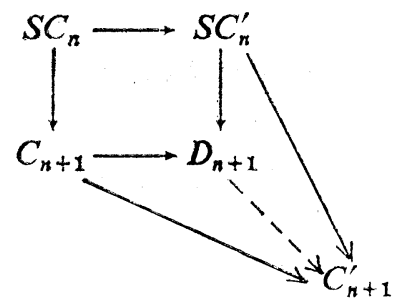

Proof. We have to check the axioms CM1-CM5 of a closed model category.

CM 1: Ps $\mathscr{C}$ is closed under finite limits and colimits. 
If $\left\{C^{i}\right\}$ is a finite diagram in Ps $\mathscr{C}$, we construct $\lim C^{i}$ and colim $C^{i}$ degreewise, i.e. $\left(\lim C^{i}\right)_{n}=\lim C_{n}^{i}$ etc. By assumption the canonical morphism colim $S C_{n}^{i} \rightarrow$ $S\left(\operatorname{colim} C_{n}^{i}\right)$ is an isomorphism, so we can compose the inverse of it with the obvious morphism colim $S C_{n}^{i} \rightarrow \operatorname{colim} C_{n+1}^{i}$ to get a morphism $S C_{n} \rightarrow C_{n+1}$, where $C=\operatorname{colim} C^{i}$. It is easily seen that the resulting prespectrum really is the colimit of the given diagram. The proof for limits is even easier.

CM2: If $f$ and $g$ are morphisms in Ps $\mathscr{C}$ and two of $f, g$ and $f g$ are weak equivalences, the third is one, too.

This is valid degreewise and thus by definition in Ps $\mathscr{C}$.

CM 3: The retract of a weak equivalence (fibration, cofibration) is a weak equivalence (fibration, cofibration).

For weak equivalences and fibrations we use degreewise arguments as above, and for the cofibrations we additionally need only an easy universality argument based on the definition of a pushout.

CM 4: Fibrations have the right lifting property with respect to trivial cofibrations and cofibrations have the left lifting property with respect to trivial fibrations.

Suppose we have a commutative diagram

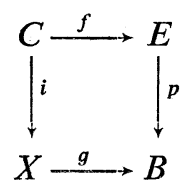

in Ps $\mathscr{C}$, where $i=\left\{i_{n}\right\}$ is a cofibration and $p=\left\{p_{n}\right\}$ a trivial fibration. The problem is to find a lifting $X \rightarrow E$, i.e. a morphism $h: X \rightarrow E$ such that $h i=f$ and $p h=g$. Degreewise a lifting $h_{n}: X_{n} \rightarrow E_{n}$ can be found in the model category $\mathscr{C}$, and thus the problem reduces to choosing the morphisms $h_{n}$ in a compatible way. In other words, we want to have the diagram

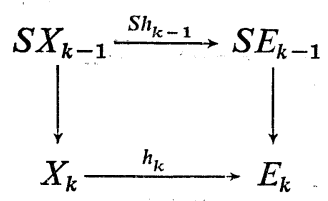

commutative for $k \geqq 1$. Suppose, then, that the liftings $h_{0}, \ldots, h_{n}$ have been defined in such a way that (2.3) is commutative, $k=1, \ldots, n$. Consider the diagram 
(2.4)

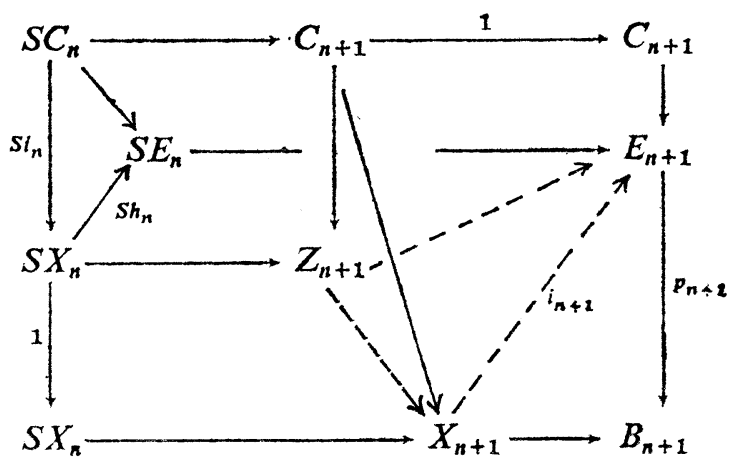

where $Z_{n+1}$ is a pushout. By assumption, the induced morphism $j_{n+1}: Z_{n+1} \rightarrow X_{n+1}$ is a cofibration; on the other hand, the morphisms $S X_{n} \rightarrow S E_{n} \rightarrow E_{n+1}$ and $C_{n+1} \rightarrow E_{n+1}$ induce a morphism $Z_{n+1} \rightarrow E_{n+1}$ such that the resulting subdiagrams are commutative by the universal property of the pushout. Thus by CM 4 (applied in $\mathscr{C}$ ) we have a lifting $h_{n+1}: X_{n+1} \rightarrow E_{n+1}$, and thus for $k=n+1$ the diagram (2.3) which is embedded as a subdiagram of (2.4) is commutative. The proof of the dual statement is similar: If $i$ is a trivial cofibration and $p$ a fibration, then $i_{n+1}$ is a weak equivalence, and furthermore $S i_{n}$ is a trivial cofibration. Thus $j_{n+1}$ is a trivial cofibration ([5], Lemma 1.2), and as $p_{n+1}$ is a fibration the lifting exists.

CM 5: Any morphism $f$ can be factorized as $f=p i$, where $p$ is a fibration and $i$ a cofibration and either $p$ or $i$ is a weak equivalence.

Of course the factorization can be performed degreewise, but the problem of compatibility remains. In addition, a degreewise cofibration need not be a cofibration. Let $f: C \rightarrow D$ be a morphism in Ps $\mathscr{C}$. We start by factoring $f_{0}=p_{0} i_{0}$, where $i_{0}: C_{0} \rightarrow X_{0}$ is a cofibration and $p_{0}: X_{0} \rightarrow D_{0}$ is a trivial fibration. Suppose that the cofibrations $i_{k}: C_{k} \rightarrow X_{k}$ and trivial fibrations $p_{k}: X_{k} \rightarrow D_{k}(k=0, \ldots, n)$ have been defined so that the diagram

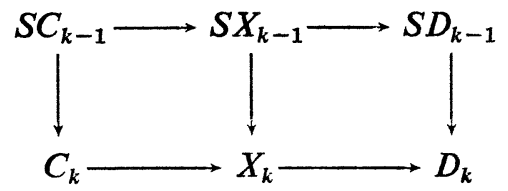

is commutative and the induced morphism $j_{k}: Z_{k} \rightarrow X_{k}$ in the pushout diagram

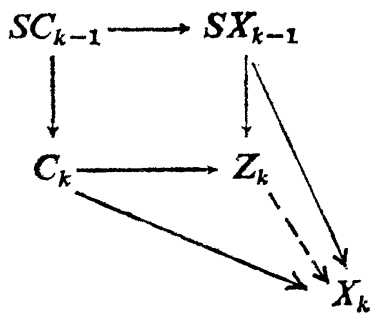


is a cofibration for $k=1, \ldots, n$. Let $C_{n+1} \rightarrow X_{n+1}^{\prime} \rightarrow D_{n+1}$ be a factorization of $f_{n+1}$ into a cofibration $i_{n+1}^{\prime}$ followed by a trivial fibration $p_{n+1}^{\prime}$. Consider the commutative diagram

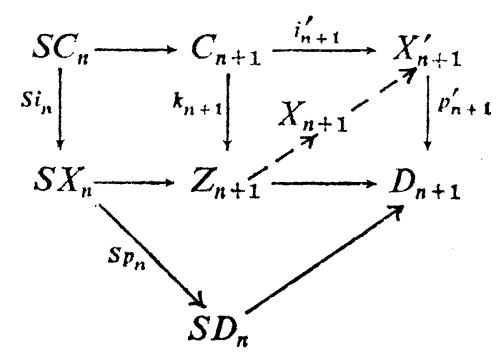

where $Z_{n+1}$ is the pushout. As $S$ preserves cofibrations, $S i_{n}$ is one. Thus $k_{n+1}$ is a cofibration and a lifting $Z_{n+1} \rightarrow X_{n+1}^{\prime}$ can be found. Factor this as $p_{n+1}^{\prime \prime} j_{n+1}$, where $j_{n+1}: Z_{n+1} \rightarrow X_{n+1}$ is a cofibration and $p_{n+1}^{\prime \prime}: X_{n+1} \rightarrow X_{n+1}^{\prime}$ is a trivial fibration. Then $i_{n+1}=j_{n+1} k_{n+1}: C_{n+1} \rightarrow X_{n+1}$ is a cofibration and $p_{n+1}=p_{n+1}^{\prime} p_{n+1}^{\prime \prime}$ is a trivial fibration, and the diagram shows that the conditions assumed for $k=0, \ldots, n$ hold for $k=n+1$.

If $i$ is to be a trivial cofibration and $p$ a fibration, the induction proceeds similarly. Now $f_{n+1}$ must be factored into a trivial cofibration $i_{n+1}^{\prime}$ followed by a fibration $p_{n+1}^{\prime}$. Then by CM 4 we find a lifting $S X_{n} \rightarrow X_{n+1}^{\prime}$, and applying the universal property of the pushout we obtain a lifting $Z_{n+1} \rightarrow X_{n+1}^{\prime}$. This is a weak equivalence ([5], Lemma 1.2), and thus $k_{n+1}$ is one, too, by CM 2. Now we factor the morphism $Z_{n+1} \rightarrow X_{n+1}^{\prime}$ as above with the difference that $j_{n+1}$ is a trivial cofibration, $p_{n+1}^{\prime}$ a fibration. Then $i_{n+1}=j_{n+1} k_{n+1}$ is a trivial cofibration, while $p_{n+1}=p_{n+1}^{\prime} p_{n+1}^{\prime \prime}$ is a fibration. The induced morphism $Z_{n+1} \rightarrow X_{n+1}$ is $j_{n+1}$ and thus a cofibration. The compatibility conditions can be inferred from the diagram.

2.8. Remark. A prespectrum over a closed category can be defined in a less general way than we have done. One could postulate, e.g., that $S C_{n} \rightarrow C_{n+1}$ is a cofibration. It is easy to see that every prespectrum is weakly equivalent to such a special prespectrum (which for example in the case of simplicial sets is the usual one). Thus the added generality does not change the homotopy theory of prespectra. 


\section{The dual of a model category with endofunctor}

If $\mathscr{C}$ is a closed model category, the dual category $\mathscr{C}^{\circ}$ can be given a natural closed category structure in the following way:

i) $f^{\circ} \in \mathscr{C}^{\circ}$ is a fibration if $f \in \mathscr{C}$ is a cofibration,

ii) $f^{\circ}$ is a cofibration if $f$ is a fibration,

iii) $f^{\circ}$ is a weak equivalence if $f$ is one.

The proof of this fact is based on the obvious self-duality of the axioms of a closed model category.

Now suppose $\mathscr{C}$ is equipped with an endofunctor $S: \mathscr{C} \rightarrow \mathscr{C}$ and that the functor $\Omega: \mathscr{C} \rightarrow \mathscr{C}$ is a right adjoint to $S$. Then $S$ preserves colimits and $\Omega$ preserves limits and thus $\Omega^{\circ}: \mathscr{C}^{\circ} \rightarrow \mathscr{C}^{\circ}$ preserves colimits. By Proposition 2.1, Ps $\mathscr{C}$ is a closed model category if $S$ preserves cofibrations and trivial cofibrations. Moreover these conditions suffice to give Ps $\left(\mathscr{C}^{\circ}\right)$ the structure of a closed model category. This is an easy consequence of the following:

3.1. Lemma. Let $\mathscr{C}$ be a closed model category with endofunctor $S: \mathscr{C} \rightarrow \mathscr{C}$ such that

i) $S$ preserves cofibrations and trivial cofibrations,

ii) there exists a right adjoint $\Omega$ to $S$.

The $\Omega$ preserves fibrations and trivial fibrations.

Proof. Let $p: X \rightarrow Y$ be a fibration. By the properties of a closed model category, it is enough to show that $\Omega(p)$ has the right lifting property with respect to trivial cofibrations. Let $i: A \rightarrow B$ be such a trivial cofibration. Consider the commutative diagram

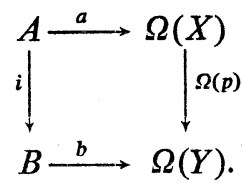

By applying $S$ and composing with the end adjunction we obtain the commutative diagram

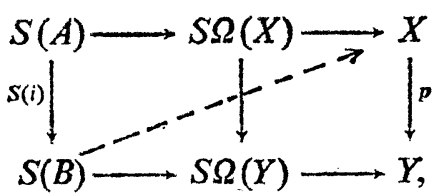

where $S(i)$ is a trivial cofibration by assumption i). Thus the dotted arrow $f: S B \rightarrow X$ can be filled in to give a lifting. Consider its adjoint morphism $f^{*}: B \rightarrow \Omega S B \rightarrow \Omega X$. The triangle 


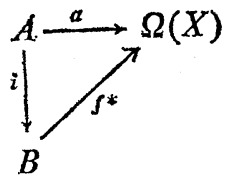

is commutative, as can be seen when it is embedded in the larger diagram

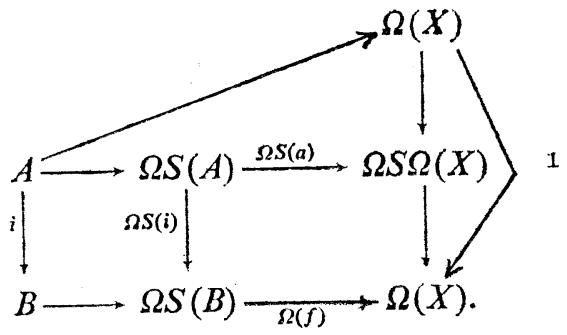

Next we have to prove that the triangle

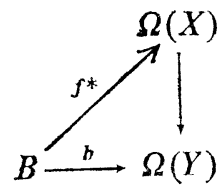

is commutative. Now $\Omega(p) f^{*}=(B \rightarrow \Omega S B \stackrel{\Omega f}{\longrightarrow} \Omega X \stackrel{\Omega p}{\longrightarrow} \Omega Y)$ and $\Omega(p) \Omega(f)=$ $(\Omega S B \stackrel{\Omega S b}{\longrightarrow} \Omega S \Omega Y \rightarrow \Omega Y)$, so we have to prove that the following diagram is commutative:

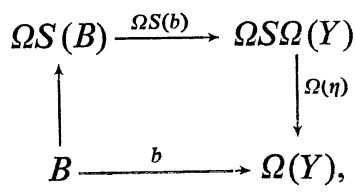

where $\eta$ is the end adjunction. But this is seen if the diagram is augmented by the front adjunction $\Omega Y \rightarrow \Omega S \Omega Y$. The proof is similar if $p$ is a trivial fibration.

Now suppose $(\mathscr{C}, S)$ satisfies the conditions of Lemma 3.1. Consider the categories Ps $\left(\mathscr{C}^{\circ}\right)$ and tow- $\mathscr{C}$. The former has as objects prespectra $\left(\Omega X_{n} \rightarrow X_{n+1}\right)$ of $\mathscr{C}^{\circ}$, i.e. sequences $\left(\Omega X_{n} \leftarrow X_{n+1}\right)$ of morphisms in $\mathscr{C}$. By adjointness these correspond to sequences $\left(S X_{n+1} \rightarrow X_{n}\right)$. Thus by defining $F\left(\Omega X_{n} \rightarrow X_{n+1}\right)=\mathscr{Y}$, where $Y_{n}=S^{n} X_{n}$, and correspondingly for morphisms, we obtain a functor $F$ : Ps $\left(\mathscr{C}^{\circ}\right) \rightarrow(\text { tow- } \mathscr{C})^{\circ}$. On the other hand we can define a functor $G:(\text { tow- } \mathscr{C})^{\circ} \rightarrow \operatorname{Ps}\left(\mathscr{C}^{\circ}\right)$ which for objects is given by $G(\mathscr{X})=\left(\Omega\left(\Omega^{n} X_{n}\right) \rightarrow \Omega^{n+1} X_{n+1}\right)$.

3.8. Proposition. The functor $F: \operatorname{Ps}\left(\mathscr{C}^{\circ}\right) \rightarrow(\text { tow- } \mathscr{C})^{\circ}$ is a left adjoint to $G:(\text { tow }-\mathscr{C})^{\circ} \rightarrow \operatorname{Ps}\left(\mathscr{C}^{\circ}\right)$. If $S$ and $\Omega$ preserve weak equivalences, $F$ and $G$ induce an adjoint couple

$$
\mathrm{Ho}\left(\operatorname{Ps}\left(\mathscr{C}^{\circ}\right)\right) \rightleftharpoons \mathrm{Ho}(\text { tow }-\mathscr{C})^{\circ} \text {. }
$$


Proof. If $S$ and $\Omega$ preserve weak equivalences, $F$ and $G$ do, too. Thus we obtain induced functors Ho $F$ and Ho $G$, which, by standard arguments used in the theory of categories of fractions, are adjoint if $F$ and $G$ are. Thus we have to prove the adjointness of $F$ and $G$.

Let $\eta: 1_{\mathscr{C}} \rightarrow \Omega S, \varphi: S \Omega \rightarrow 1_{\mathscr{C}}$ be the natural transformations of the adjoint couple $(S, \Omega)$. Then, as is well known, $\left(S^{n}, \Omega^{n}\right)$ is an adjoint couple with adjunction morphisms

defined recursively by

$$
\begin{aligned}
& e_{n}(X): X \rightarrow \Omega^{n} S^{n} X \\
& f_{n}(X): S^{n} \Omega^{n} X \rightarrow X
\end{aligned}
$$

$$
\begin{gathered}
e_{0}(X)=1_{X}, \\
e_{n+1}(X)=\Omega^{n}\left(\eta\left(S^{n} X\right)\right) e_{n}(X): X \rightarrow \Omega^{n} S^{n} X \rightarrow \Omega^{n+1} S^{n+1} X, \\
f_{0}(X)=1_{X}, \\
f_{n+1}(X)=f_{n}(X) S^{n}\left(\varphi\left(\Omega^{n} X\right)\right): S^{n+1} \Omega^{n+1} X \rightarrow S^{n} \Omega^{n} X \rightarrow X .
\end{gathered}
$$

It is clear that $e_{n}$ and $f_{n}$ are natural in $X$.

Now we define a transformation $H: 1_{\mathrm{Ps}\left(\mathscr{C}^{\circ}\right)} \rightarrow G F$ by

$$
H\left(\left(X_{k}\right)\right)_{n}=e_{n}\left(X_{n}\right): X_{n} \rightarrow \Omega^{n} S^{n} X_{n} .
$$

Degreewise this is natural, so we only have to prove that we obtain a morphism in Ps $\left(\mathscr{C}^{\circ}\right)$ in this way. This amounts to proving the commutativity of the diagram

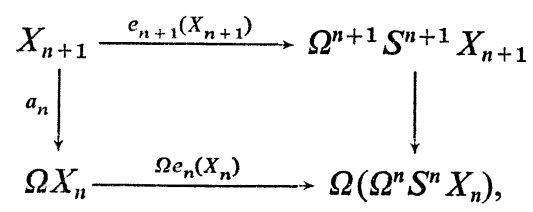

where $a_{n}$ is the structural map of the prespectrum and the unmarked morphism is the composite $\Omega^{n+1} S^{n+1} X_{n+1} \stackrel{\Omega^{n+1} S^{n+1}\left(a_{n}\right)}{\longrightarrow} \Omega^{n+1} S^{n+1} \Omega X_{n} \stackrel{\Omega^{n+1} S^{n} \varphi\left(X_{n}\right)}{\longrightarrow} \Omega^{n+1} S^{n} X_{n}$.

Thus it is enough to prove the commutativity of the diagram

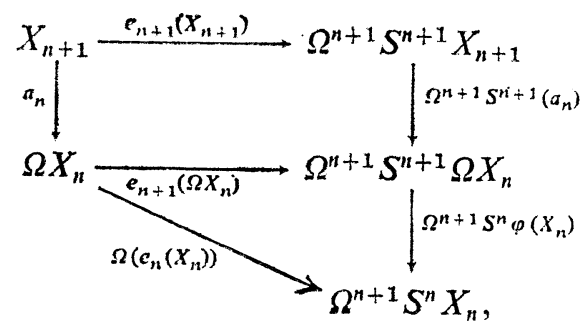

and as the square is commutative by the naturality of $e_{n+1}$, it is enough to prove the commutativity of the triangle. For $n=0$ this is evident, and using the naturality of $\eta$ one proves the general case by induction. 
We now define a natural transformation $\Phi: F G \rightarrow 1_{(\text {tow- } ⿻)^{\circ}}$ by

$$
\Phi(\mathscr{Y})_{n}=f_{n}\left(Y_{n}\right): S^{n} \Omega^{n} Y_{n} \rightarrow Y_{n}
$$

for a tower $\mathscr{Y}: \ldots \rightarrow Y_{n+1} \rightarrow Y_{n} \rightarrow \ldots$. That $\Phi$ is a well-defined natural transformation is proved as above. The identities

$$
(G(\mathscr{Y}) \stackrel{H(G(\mathscr{y}))}{\longrightarrow} G F G(\mathscr{Y}) \stackrel{G(\Phi(\mathscr{y}))}{\longrightarrow} G(\mathscr{Y}))=1_{G(\mathscr{y})}
$$

and

$$
\left(F\left(\left(X_{n}\right)\right) \stackrel{F\left(H\left(\left(X_{n}\right)\right)\right)}{\longrightarrow} F G F\left(\left(X_{n}\right)\right) \stackrel{\Phi\left(F\left(\left(X_{n}\right)\right)\right)}{\longrightarrow} F\left(\left(X_{n}\right)\right)\right)=1_{F\left(\left(X_{n}\right)\right)}
$$

follow from the adjointness of $S^{n}$ and $\Omega^{n}$.

3.15. Example. If $\mathscr{C}$ is the category of simplicial sets, $S$ the simplicial suspension and $\Omega=\omega$ its adjoint as defined in [4], $\Omega S=\omega S=1_{\mathscr{C}}$ and thus $\operatorname{Ps}\left(\mathscr{C}^{\circ}\right)$ can in this case be embedded as a full subcategory of (tow- $\mathscr{C})^{\circ}$.

\title{
References
}

[1] BAUER, F. W.: Boardman's category and the process of categorical stabilization. - Math. Z. 130, 1973, 95-106.

[2] Edwards, D. A., and H. M. Hastings: Čech and Steenrod homotopy theories with applications to geometric topology. - Lecture Notes in Mathematics 542, Springer-Verlag, BerlinHeidelberg-New York, 1976.

[3] Grossman, J. W.: A homotopy theory of pro-spaces. - Trans. Amer. Math. Soc. 201, 1975, $161-175$.

[4] KAN, D. M.: Semisimplicial spectra. - Illinois J. Math. 7, 1963, 463-478.

[5] MALM, K.: Remarks on the closed model category structure of simplicial spectra. - Ann. Acad. Sci. Fenn. Ser A I Math. 3, 1977, 257-266.

[6] MaLm, K.: A model category structure for stabilized categories. - Reports of the Department of Mathematics, University of Helsinki, 1977, 1-19.

\author{
University of Helsinki \\ Department of Mathematics \\ SF-00100 Helsinki \\ Finland
}

Received 22 January 1987 